\title{
Sur l'unité de l'Aurignacien ancien dans le Sud-Ouest de la France: la production des lames et des lamelles
}

\author{
JEAN-GuILlaUme Bordes ${ }^{1}$ \\ JACQUES TIXIER ${ }^{2}$
}

RÉSUMÉ

Dans le Sud-Ouest de la France, l'Aurignacien ancien "classique», à sagaies à base fendue, est un technocomplexe caractérisé par une très forte unité technique des productions laminaire et lamellaire. Cet article est une contribution à la caractérisation des concepts et des modalités qui régissent la production de ces deux types de supports.

Nous nous appuyons essentiellement sur le matériel de Corbiac-Vignoble II, atelier de taille où une matière première "parfaite" a permis aux tailleurs

aurignaciens de s'exprimer quasiment sans contraintes, illustrant ainsi au mieux les concepts de taille sous-jacents à toutes les séries d'Aurignacien ancien récemment étudiées.

Lames et lamelles sont toujours produites à la percussion directe au percuteur tendre. Les schémas de production de ces deux types de supports sont disjoints.

- Les lames de fort gabarit sont produites à partir de blocs gérés selon un

\section{RESUMEN}

En el suroeste francés, el Auriñaciense "clásico", de azagayas de base hendida, es un tecnocomplejo caracterizado por una unidad técnica muy marcada en cuanto a la producción de hojas y de hojitas. Este articulo es una contribución a la caracterización de los conceptos y las modalidades que rigen la producción de estos dos tipos de soportes.

Nos basamos en el material de Corbiac-Vignoble II, taller de talla donde una materia prima "ideal» ha permitido a los talladores auriñacienses expresarse, casi sin limitaciones, ilustrando asi todas las series del Auriñaciense antiguo recientemente estudiadas.

Las hojas y las hojitas son siempre producidas mediante percusión directa con percutor blando. Los esquemas de producción de estos dos tipos de soportes son siempre separados.

- Las hojas de grandes dimensiones son realizadas a partir de nódulos gestionados

\footnotetext{
1 PACEA - IPGQ - UMR 5199; Université Bordeaux 1; 33405 Talence cedex;

jg.bordes@ipgq.u-bordeaux1.fr

${ }^{2}$ Les Places, 11 Clos des Combels; 46090 Pradines; fax: 0033 (0)5.65.53.96.25
} 
schéma unipolaire "frontal", dont la mise en forme est toujours minimale.

- Les lamelles sont produites à partir de nucléus sur éclat de type "grattoir caréné". Leur profil est indifféremment rectiligne ou courbe, mais jamais tors.

Cette structuration contribue à la définition d'un système techno-économique traduisant une grande circulation des objets ou des personnes, et donc des idées. Cette définition explique à la fois

l'unité et la visibilité dans le registre archéologique de l'Aurignacien ancien.

La répartition géographique de ce technocomplexe reste à définir par des analyses similaires. Elle n'est probablement pas aussi étendue que les travaux basés uniquement sur une typologie morphologique des outillages ont pu le laisser croire.

\section{MOTS CLÉFS}

Aurignacien, Ancien, lames, lamelles, Corbiac-Vignoble II. mediante un esquema unipolar "frontal" $y$ cuya mise en forme es siempre mínima.

- Las hojitas se producen a partir de núcleos sobre lasca de tipo «raspador carenado". Su perfil es rectilíneo o curvo, pero jamás presenta torsión.

Esta estructuración contribuye a la definición de un sistema tecno-económico que refleja una gran circulación de objetos $y$ de personas, $y$, por supuesto, de ideas. Esta definición explica a la vez la unidad y la visibilidad del registro arqueológico del Auriñaciense antiguo. Queda por definir la distribución geográfica de este tecnocomplejo mediante estudios similares, ya que no se pueden entender únicamente a partir de los estudios basados en la tipología morfológica de los útiles como se ha pensado hasta ahora.

\section{PALABRAS CLAVE}

Auriñaciense Antiguo, hojas, hojitas, Corbiac-Vignoble II.

\section{INTRODUCTION}

Depuis la reconnaissance de l'Aurignacien, sa phase ancienne, à sagaies à base fendue, stade «l» de Peyrony $(1933,1934)$, est la plus clairement identifiée. Cela tient probablement au fait que, comme le souligne D. de Sonneville-Bordes dans sa synthèse sur le Sud-Ouest de la France (1960), les occupations attribuables à cet épisode de l'Aurignacien sont nombreuses, les niveaux concernés facilement identifiables en stratigraphie (forte anthropisation des niveaux par de l'ocre ou des charbons) et riches en objets caractéristiques. L'analyse typologique quantifiée des industries lithiques (Sonneville-Bordes, id.) a d'ailleurs pleinement confirmé la classification de Peyrony pour cette phase de l'Aurignacien: les outillages associés aux sagaies à base fendue dans le Sud-Ouest de la France se reconnaissent toujours sans ambiguïté, et possèdent de nombreux caractères communs.

Pour la majorité des chercheurs, ces caractères, en fort contraste avec le Châtelperronien sous-jacent, ont contribué à faire de l'Aurignacien ancien la marque de l'arrivée de l'homme anatomiquement moderne en Europe, voire en Eurasie (e. g. Harrold et Otte, 2001; Kozlowski et Otte, 2000; Mellars, 1996, 2004). Si ce modèle est aujourd'hui contredit par les données (cf. infra), l'Aurignacien ancien reste une phase repère du Paléolithique supérieur ancien, qui s'individualise 
par son unité. C'est cette unité que nous voulons ici contribuer à documenter, à travers une description des schémas de productions de lames et de lamelles. L'objectif est de poser les premiers jalons d'une caractérisation techno-économique de l'Aurignacien ancien, afin d'en mieux comprendre le fonctionnement socioéconomique.

Les nombreuses études récentes sur des séries attribuables à l'Aurignacien ancien dans le Sud-Ouest de la France ne font que confirmer son unité technique. Notre objectif ici est d'étayer cette unité par la description d'une série qui nous semble particulièrement représentative des "manières de voir» et "manières de faire" des tailleurs aurignaciens anciens: Corbiac-Vignoble II. En effet, ce site de plein air de la région de Bergerac (Dordogne), atelier de taille situé près de gîtes d'une matière première idéale, a permis aux artisans d'exprimer sans contrainte leur savoir-faire. De plus, la quasi-absence des phases de retouche et utilisation des outils dans ce site conduit à une meilleure visibilité du débitage, non brouillé par les opérations de réduction successives des supports, nucléus et déchets que l'on rencontre habituellement dans les sites dits d'habitat, c'est à dire ayant livré de nombreux outils et autres témoignages d'un large registre d'activités.

Mais avant d'aborder cette description, nous effectuons sous forme de bilan synthétique et probablement encore très partiel, quelques autres éléments de caractérisation du technocomplexe «Aurignacien ancien du Sud-Ouest de la France »: la chronologie, l'outillage, la mobilité.

\section{LA SEQUENCE AURIGNACIENNE EN AQUITAINE}

Depuis Peyrony (1933), de nombreux modèles ont été élaborés sur la base de l'analyse typologique et des matières premières. II n'est pas impossible que l'analyse technologique conduise à retrouver pro parte l'un ou l'autre de ces modèles. Cependant, il nous semble plus prudent de s'en tenir aux séries dont la validité a été réévaluée par une analyse taphonomique, et qui permettent actuellement de distinguer trois épisodes principaux pour l'Aurignacien en Aquitaine. Encadrant chronologiquement l'Aurignacien ancien on distingue:

- Une phase récente, à nucléus à lamelles de types "grattoirs à museau», «burins busqués" et autres «burins des vachons" destinés à produire surtout de petites lamelles torses, regroupe l'Aurignacien "Il» à "IV" de D. Peyrony (id.). Si certains de ces schémas de production lamellaire sont aujourd'hui solidement documentés (Lucas 1997, 2000; Chiotti 1999, 2000; Bordes et Lenoble, 2002), ce n'est pas le cas pour la production laminaire qui, dans une première analyse, semble proche de ce qui est connu pour l'Aurignacien ancien. Cette phase est la moins bien connue d'un point de vue chronostratigraphique, et il semble hautement probable que l'on puisse, à l'avenir, y effectuer des subdivisions chronologiques. Mais les ensembles concernés sont relativement peu nombreux, particulièrement pour les stades «III» et «IV», s'ils existent. 
- Précédant l'Aurignacien ancien, il existe une industrie caractérisée par la continuité des débitages laminaire et lamellaire, et la production de grandes lamelles de profil plutôt rectiligne. Cette industrie est à rapprocher d'une part de l'Aurignacien "archaïque» tel qu'il est défini dans le pourtour méditerranéen (J.-G. Bon, 2002, pour une synthèse), et d'autre part du Châtelperronien (Bordes, 2002 et sous presse). Dans le Sud-Ouest de la France, elle a été retrouvée à Dufour (Corrèze; lors d'un travail en collaboration avec F. Bon), à la Chaise (Charente; F. Bordes, 1958; Demars et Laurent, 1989, p. 103), à La Ferrassie niveau E' (travail en cours par J.G.B.), au Piage (Lot; J.-G. Bordes, 2002), dans les Pyrénées occidentales et les Cantabres (Arrizabalaga et al., à paraître). L'analyse comparative de ces séries, qui reste à poursuivre, impose d'ores et déjà de repenser les bases des modèles concernant la transition du Paléolithique moyen au Paléolithique supérieur (J.-G. Bordes, sous presse).

Les caractères communs à l'ensemble de ces industries sont: obtention des supports recherchés - lames et lamelles - par percussion directe au percuteur tendre, absence de production d'éclats destinés à être retouchés, nucléus majoritairement unipolaires, pièces carénées, retouche parfois directe mais plus souvent inverse, courte et semiabrupte, que portent la plupart du temps des supports lamellaires (retouche de «type Dufour», Brézillon, 1968, p. 114).

\section{L'OUTILLAGE RETOUCHE A L'AURIGNACIEN ANCIEN EN AQUITAINE: NORMES ET VARIATIONS}

Nous excluons de ces observations les pièces carénées, qui seront traitées en tant que nucléus dans la description des schémas opératoires (cf. Infra). Même si, parfois, ces pièces ont aussi pu être utilisées comme outils leur statut de nucléus nous semble plus pertinent pour définir la structuration techno-économique des industries de l'Aurignacien.

\subsection{Outils sur lame}

Avant les types d'outils, c'est d'abord un type de retouche qui caractérise l'Aurignacien ancien: la retouche "aurignacienne" (Sonneville-Bordes, 1960, p. 20; Brézillon, 1968, p. 113). Cette retouche écailleuse est bien différente de la retouche Quina en ce sens qu'elle ne diminue pas l'angle de coupe mais qu'au contraire, elle l'augmente. Plutôt que d'un façonnage spécifique, cette retouche semble résulter du ravivage des supports: ceux-ci sont en effet la plupart du temps fortement réduits par les opérations de retouche successives, et semblent avoir porté des types d'outils différents au cours de leur fonctionnement (J.-G. Bordes, 2002).

L'outillage sur lame est toujours dominé par les grattoirs et les pièces à retouche latérale. La variabilité typologique s'exprime au niveau de la représentation relative des pièces esquillées et des burins. 
La variabilité de la proportion de pièces esquillées dans l'outillage semble liée à l'importance de certaines activités dans les sites d'une part et à l'éloignement de bonnes sources de matières premières d'autre part (Le Brun-Ricalens, 1989).

II est plus difficile de proposer des hypothèses sur la (ou les) cause(s) de la variabilité de la proportion de burins dans l'outillage. Celle-ci a fondé la définition de deux faciès (Aurignacien ancien de type Castanet: pauvre en burins; de type Lartet-Ferrassie: plus riche en burins), dont it n'est toujours pas possible de déterminer s'ils ont une signification chronologique, économique ou fonctionnelle (Sonneville-Bordes, 1960).

\subsection{Outils sur lamelle}

La rareté des lamelles retouchées à l'Aurignacien ancien, surtout lorsqu'on la compare au souvent grand nombre de pièces carénées, incite à penser que la majorité des supports lamellaires ont été utilisés bruts, ou abandonnés sur des sites que l'archéologie n'a pas encore décelés.

Le type dominant est toujours la lamelle Dufour, c'est-à-dire qui possède une retouche le plus souvent inverse (sur le bord droit) et parfois alterne, courte, semiabrupte. Parmi ces objets, il existe dans certains sites une forte proportion d'éléments mésiaux de dimensions assez comparables, et parfois tronqués (Bon, 2002, J.-G. Bordes, sous presse). Les supports retouchés montrent des dimensions comprises entre 2 et $4 \mathrm{~cm}$ de long, pour 5 à $10 \mathrm{~mm}$ de large. Ils sont de profil indifféremment courbe ou rectiligne, mais jamais tors.

\subsection{Les matières premières lithiques à l'Aurignacien ancien en Aquitaine}

L'importance dans l'outiliage des matières premières non locales est une caractéristique classique de cette phase de l'Aurignacien (e.g. Demars, 1994). La découverte récente de matériaux d'origine nord-pyrénéenne et charentaise dans nombre de ces gisements modifie notre vision de la mobilité de ces groupes (J.-G. Bordes et al., sous presse, Le Brun-Ricalens et Séronie-Vivien, 2004). Plus que pour tout autre technocomplexe du Paléolithique supérieur, les séries attribuables à l'Aurignacien se caractérisent aussi par la variété des matières premières exploitées, locales ou lointaines (obs. personnelles et A. Morala, com. orale).

Que ce soit le témoignage d'échanges entre groupes, ou encore d'une circulation des groupes eux-mêmes, ces transports de silex sur de longues distances sont des marqueurs de structures sociales basées sur la mobilité. 


\section{CORBIAC-VIGNOBLE //}

II s'agit d'un site de plein air de la région de Bergerac, fouillé de 1987 à 1989 sous la direction de J. Tixier. II est situé près d'une source d'excellente matière première lithique, disponible en grande quantité. La fouille a concerné plus de 69 mètres carrés, et permis d'exhumer environ 7500 artefacts de plus de $3 \mathrm{~cm}$, représentant entre 170 et $250 \mathrm{~kg}$ de matériel. Le tamisage systématique a été accompagné de la récolte exhaustive des artefacts de plus de $2 \mathrm{~mm}$ de dimension maximale, et a permis de recueillir plus de $6 \mathrm{~kg}$ de petites esquilles. Les techniques de fouilles choisies ont permis, entre autres, de réaliser un plan photographique exact, grâce à un harnais spécial (brevet déposé, voir Tixier, 1991) de la partie sommitale non perturbée des pièces en place, et à partir duquel un plan a pu être construit (Fig. 1). Au dessous, la récolte du volume des pièces a été effectuée par décapages successifs et surfaces modulées (mètre carré, quart, seizième) suivant la densité plus ou moins grande des pièces, en vue d'établir une

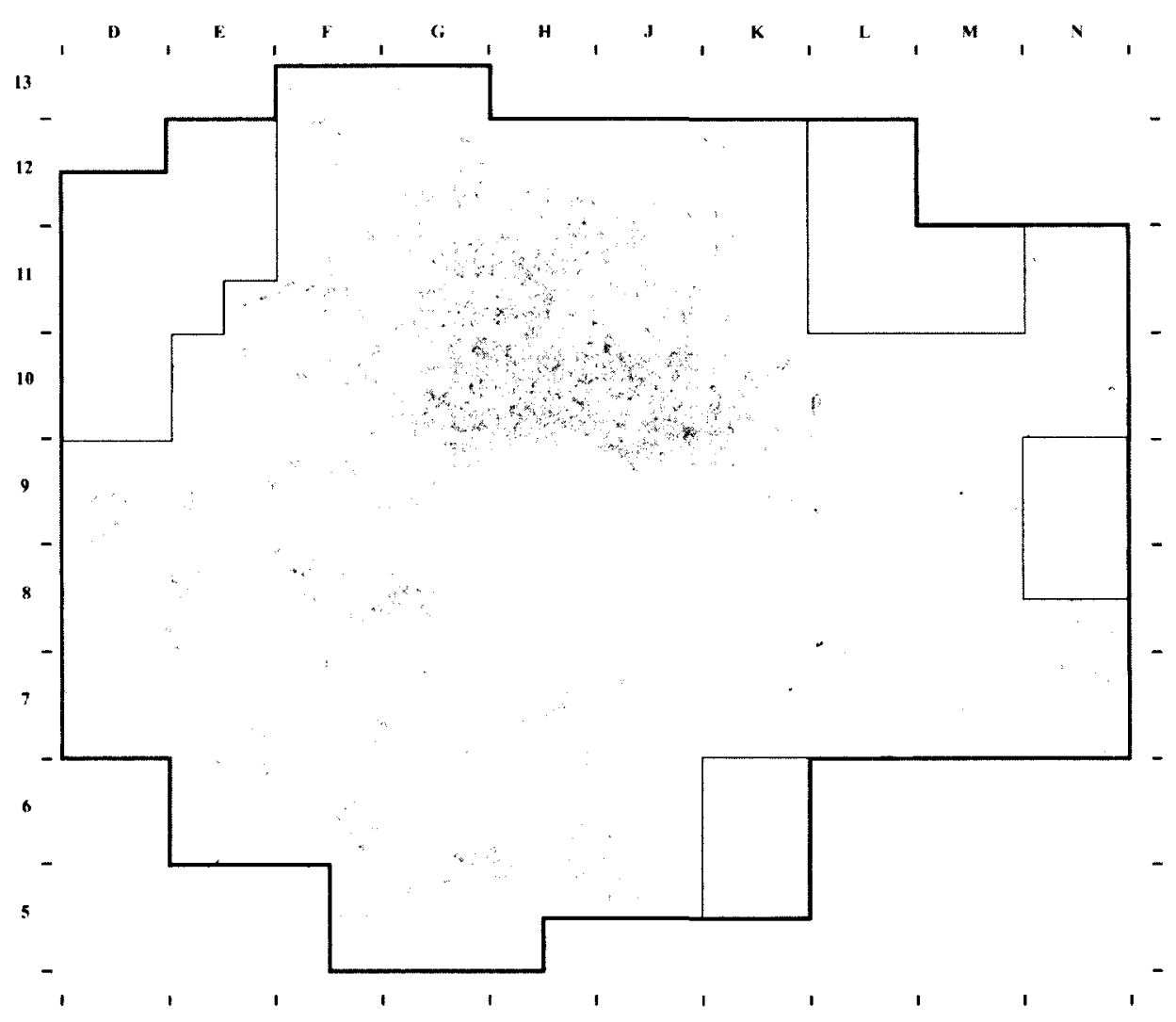

Figure 1. Corbiac-Vignoble II, plan de la fouille (chaque graduation représente un mètre). Les zones en grisé ont été fouillées sans prises de coordonnées. 
«répartition technologique». Une première analyse technologique a montré que le site était un atelier de taille de lames et de lamelles, attribuable à l'Aurignacien (Tixier et Reduron, 1991). Sur la même parcelle de terrain, deux autres amas attribuables à l'Aurignacien ont été découverts (Chadelle, 1989).

La structuration spatiale évidente (Fig. 1), le très grand nombre de remontages (plus de 1000 liaisons effectuées à ce jour), et la très forte homogénéité technique du matériel lithique, seul conservé, font de ce site un instantané d'occupation. Si la répartition horizontale des vestiges semble avoir été assez bien préservée, la cryoturbation a causé des mouvements verticaux des pièces. Lors de ces déplacements, qui ont donné à la nappe de vestiges une morphologie ondulée, la pression provoquée par le gel a affecté les bords de certaines de ces pièces (cf. fig. 2 et 5). Grâce à une matière première idéale et aux nombreux remontages, les objectifs et les modalités de la taille, pleinement exprimés, peuvent être décrits très précisément. Nous n'en aborderons ici que quelques principes essentiels. La poursuite de la réalisation de remontages dans ce site pourrait permettre une évaluation plus précise du nombre de supports emportés par les artisans aurignaciens.

\subsection{Lames}

\subsubsection{Choix des blocs et mise en forme}

Le premier choix effectué par les tailleurs est celui de blocs de morphologie ne nécessitant qu'une préparation minimale pour le débitage laminaire, et dont les dimensions permettront de débiter des lames d'au moins $10 \mathrm{~cm}$ de long. Ces blocs peuvent être classés en deux catégories: les dalles et les rognons.

Les deux faces parallèles des dalles constituent les flancs des nucléus, et ne sont jamais modifiés. Dans ce cas, la mise en forme est réduite à la confection sans grand soin d'une crête à deux versants préparés par percussion directe au percuteur dur. Cette crête peut concerner seulement un bord de la dalle: il s'agira alors de la future surface de débitage. Mais elle peut s'étendre à trois bords de la dalle, le quatrième étant réservé au plan de frappe. Ce dernier, lorsqu'il n'est pas constitué d'une surface plane naturelle (diaclase), est mis en place par de grands éclats débités à partir de l'un des flancs.

La mise en forme des blocs de morphologie oblongue (rognons) est similaire, à ceci près que les premiers enlèvements laminaires, totalement puis largement corticaux, ont pour but, outre de mettre en place des nervures guides qui assurerons la poursuite du débitage, d'augmenter le cintre de la surface de débitage, afin de se rapprocher de la morphologie "dalle", aux flancs parallèles. $\mathrm{Si}$ la morphologie du bloc le nécessite, ces enlèvements sont volontiers épais, et parfois même débités au percuteur de pierre (ce sont alors des éclats laminaires). A partir de cette configuration, le débitage suit des procédures indépendantes de la morphologie initiale du bloc. 
4.1.2. Plein débitage et entretien de la morphologie du nucléus

Ces deux notions nous semblent ici pleinement liées, tant certains produits d'entretien sont effectivement exportés, au même titre que ce que nous nommons les supports de plein débitage.
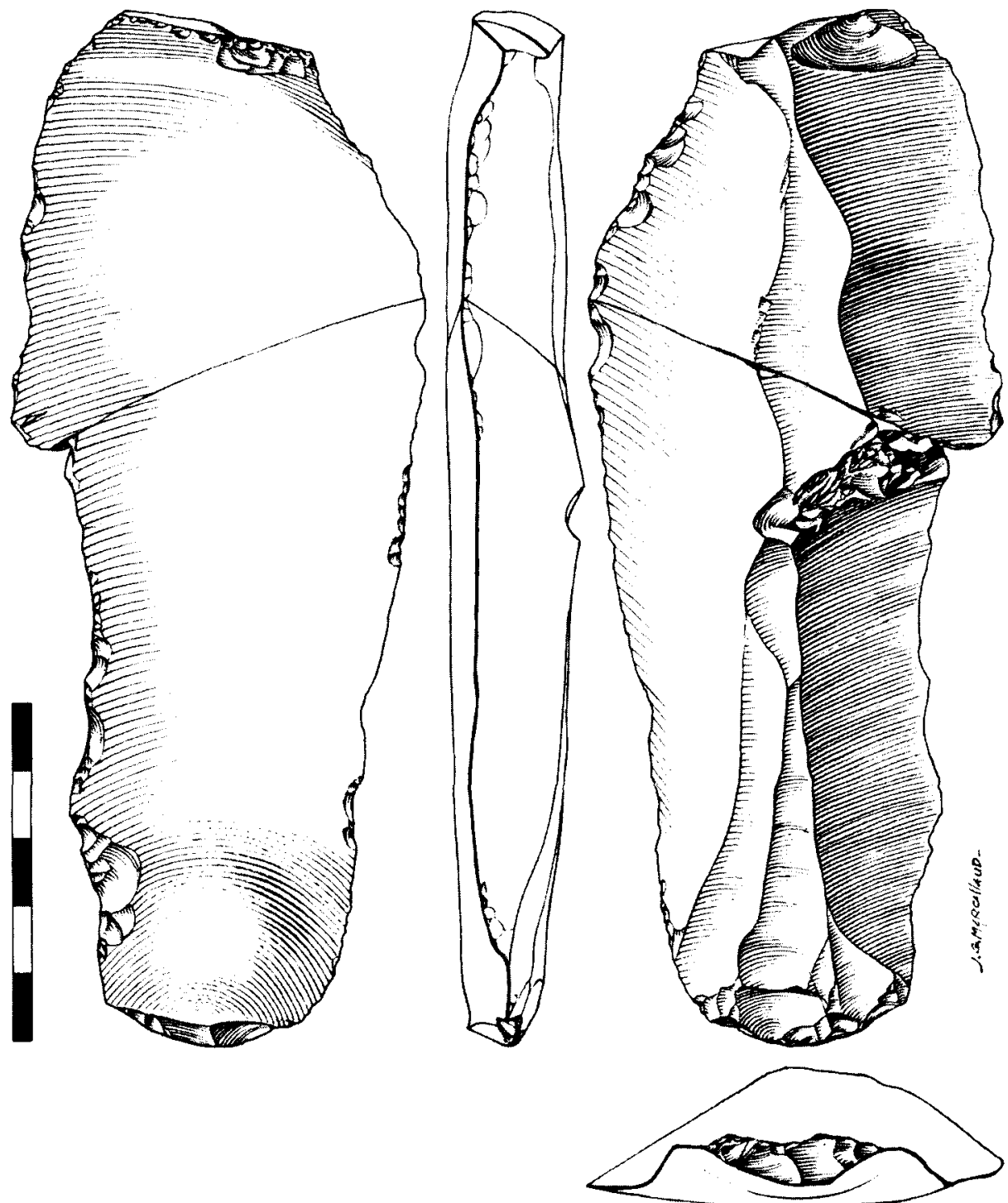

Figure 2. Corbiac-Vignoble II, lame non corticale. Remarquer l'écrasement d'une nervure proéminente résultant d'un rebroussé. Dessin J.-G. Marcillaud. 
La progression du débitage est ufrontale» (Pigeot, 1987): les enlèvements laminaires n'investissent que peu les flancs. Ces derniers restent donc globalement perpendiculaires à la surface de débitage.

Qu'elles soient au centre de la surface de débitage (lames non corticales, Fig. 2), ou à l'intersection de celle-ci avec l'un des flancs (lames de recintrage, à un pan cortical, Fig. 3a), les lames sont voulues larges et épaisses: chaque enlèvement est préparé par un facettage spécifique et abrasion au niveau du plan de frappe. Le but de cette préparation est triple: régler au mieux l'angle plan de frappe / surface de débitage, mettre en évidence le futur point d'impact, calibrer l'épaisseur des supports recherchés. Cette opération se traduira sur ces derniers par des talons facettés, voire en éperon peu dégagé.

Les lames exportées mesurent entre 20 et $10 \mathrm{~cm}$ de long, pour 5 à $2,5 \mathrm{~cm}$ de large et 7 à $15 \mathrm{~mm}$ d'épaisseur en moyenne. Elles possèdent généralement un profil courbe, surtout en partie distale. Cette courbure est souvent donnée par une augmentation de l'épaisseur de nombreux supports dans leur partie distale, qui va parfois jusqu'à l'outrepassage.

Les outrepassages accidentels sont rares: ils conduisent à un raccourcissement du nucléus préjudiciable à la poursuite du débitage. Il semble que la majorité des lames à tendance "outrepassante" soi voulue comme telle: elle permet de recréer de la courbure en partie distale du nucléus sans défigurer la surface de débitage comme a tendance à le faire une crête (disparition des nervures-guide). Le caractère intentionnel de ces enlèvements est démontré par l'angle très ouvert crée par le facettage de la zone de détachement des supports concernés: un tel angle impose une énergie accrue pour le détachement, qui aura ainsi tendance à se propager de plus en plus en profondeur dans le silex. Notons que les plus fréquentes de ces lames sont situées à l'intersection entre les surfaces de débitage et l'un des flancs: la courbure de l'outrepassage entretient donc autant le cintre que la carène distale (Fig.3a).

Un autre élément d'entretien de la surface de débitage, largement utilisé, est la crête à un versant préparé à partir de la surface de débitage, dite aussi néocrête (Pelegrin, 1995). Celle-ci est réalisée au percuteur dur, sans ménagement. Elle peut être réduite à quelques enlèvements faisant disparaître une légère concavité (Fig. 3), jusqu'à concerner l'ensemble de la surface de débitage (Fig. 4).

Une autre procédure de remise en forme de la surface de débitage est la crête à deux versants préparés, initiée à partir de la partie distale du nucléus, et qui "remonte" plus ou moins sur la surface de débitage en fonction de la localisation de la zone à corriger. Cette procédure a l'inconvénient de détruire les nervures guide du débitage laminaire, la crête produite étant de délinéation peu régulière. Elle est le plus souvent réduite à la partie distale de la surface de débitage.

Proche de la crête à un versant préparé, mais ne produisant aucun enlèvement, l'écrasement de nervures gênantes par un véritable "bouchardage" est une procédure d'entretien fréquemment employée (Fig. 2). 


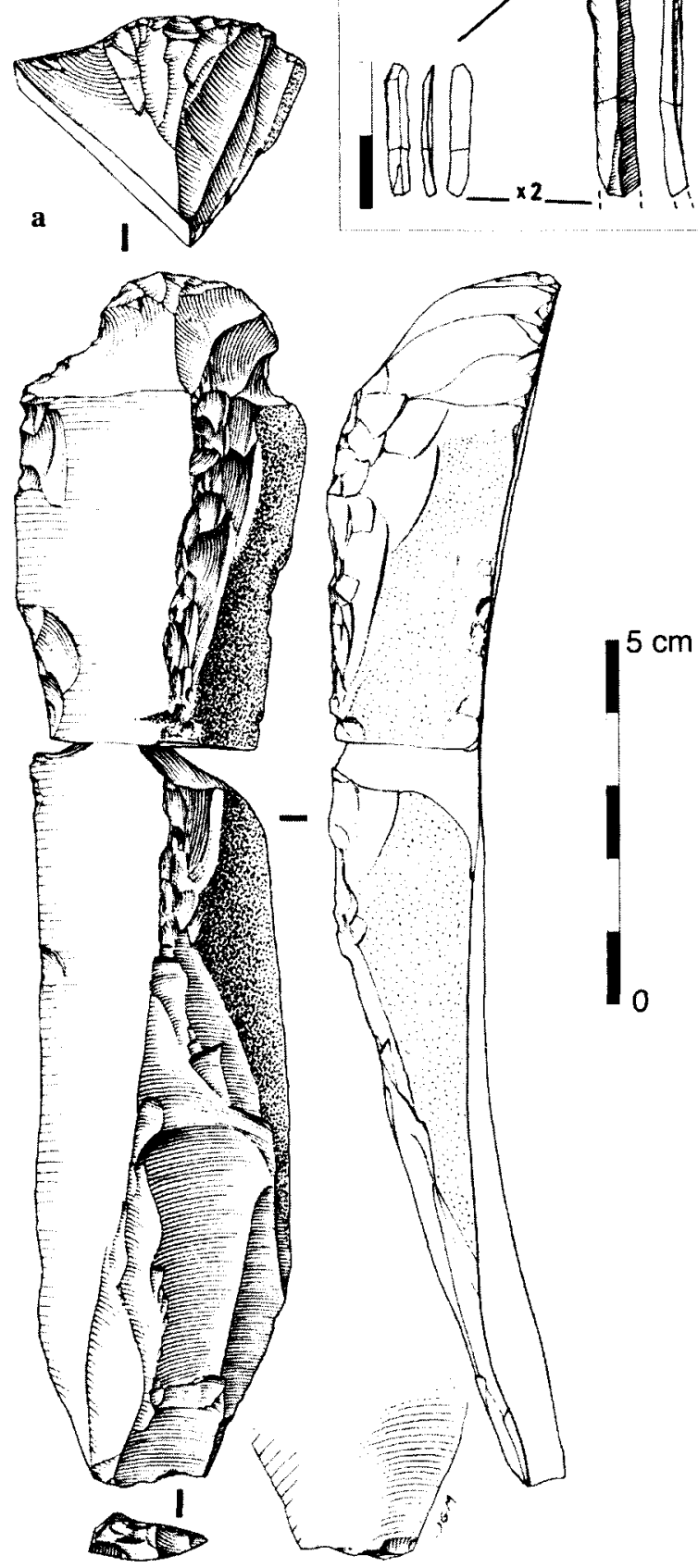

Fig. 3: A) Corbiac-Vignoble II, lame à un pan cortical, à tendance "outrepassante". Nucléus à lamelles de type "grattoir caréne". B) lamelle Dufour, silex gris du Sénonien. Dessin J.-G. Marcillaud. 
Le plan de frappe est constamment entretenu par un facettage des futurs points d'impacts. Lorsque cette procédure, répétée pour chaque enlèvement, a complètement défiguré la surface d'implantation du plan de frappe, des tablettes de ravivage, souvent épaisses, sont produites. Si le maintien d'une surface de plan de frappe tout au long du débitage reste la méthode préférée, la mise en place d'une seconde, opposée à la première, se retrouve sur certains blocs. II ne s'agit pas pour autant d'un véritable débitage bipolaire: cette deuxième surface de plan de frappe a seulement pour but de recréer une courbure distale ou de supprimer un rebroussé. Parfois aussi, le nucléus est complètement réorienté, mais le débitage reste effectué à partir d'une seule surface de plan de frappe.

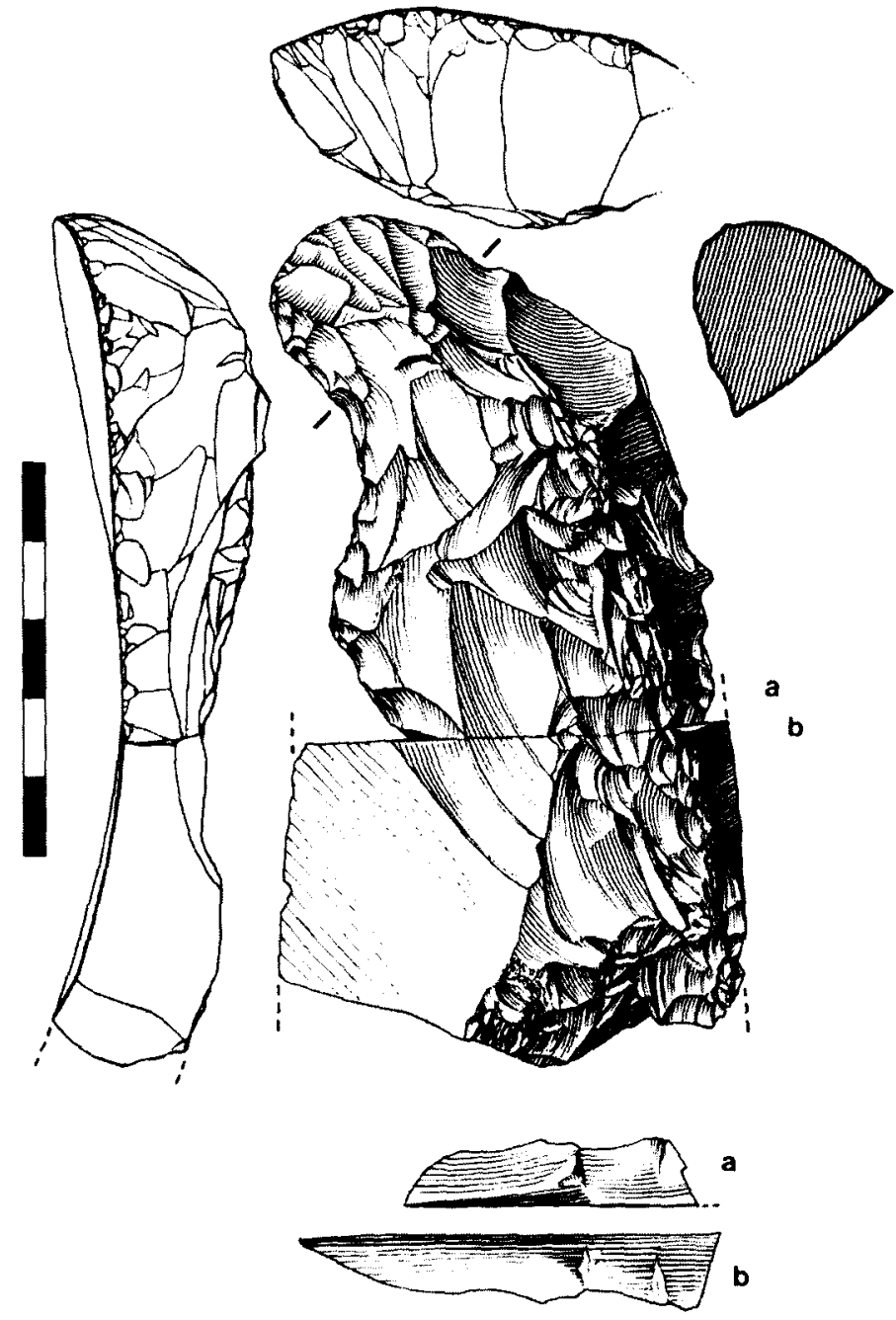

Figure 4. Corbiac-Vignoble II, partie distale d'une lame néocrête, nucléus à lamelle de type "grattoir caréné". Dessin J.-G. Marcillaud. 


\subsubsection{Abandon du débitage, abandon des supports}

L'examen des objets abandonnés sur un atelier de taille nous renseigne sur ce que les tailleurs ne voulaient pas. C'est donc, par complémentarité, une indication précieuse sur leurs objectifs. Les lames abandonnées sur les sites sont:

- les entames corticales (Fig. 5),

- les pièces cassées au débitage, dont aucun fragment aux bords réguliers ne dépasse $4-5 \mathrm{~cm}$ de long,

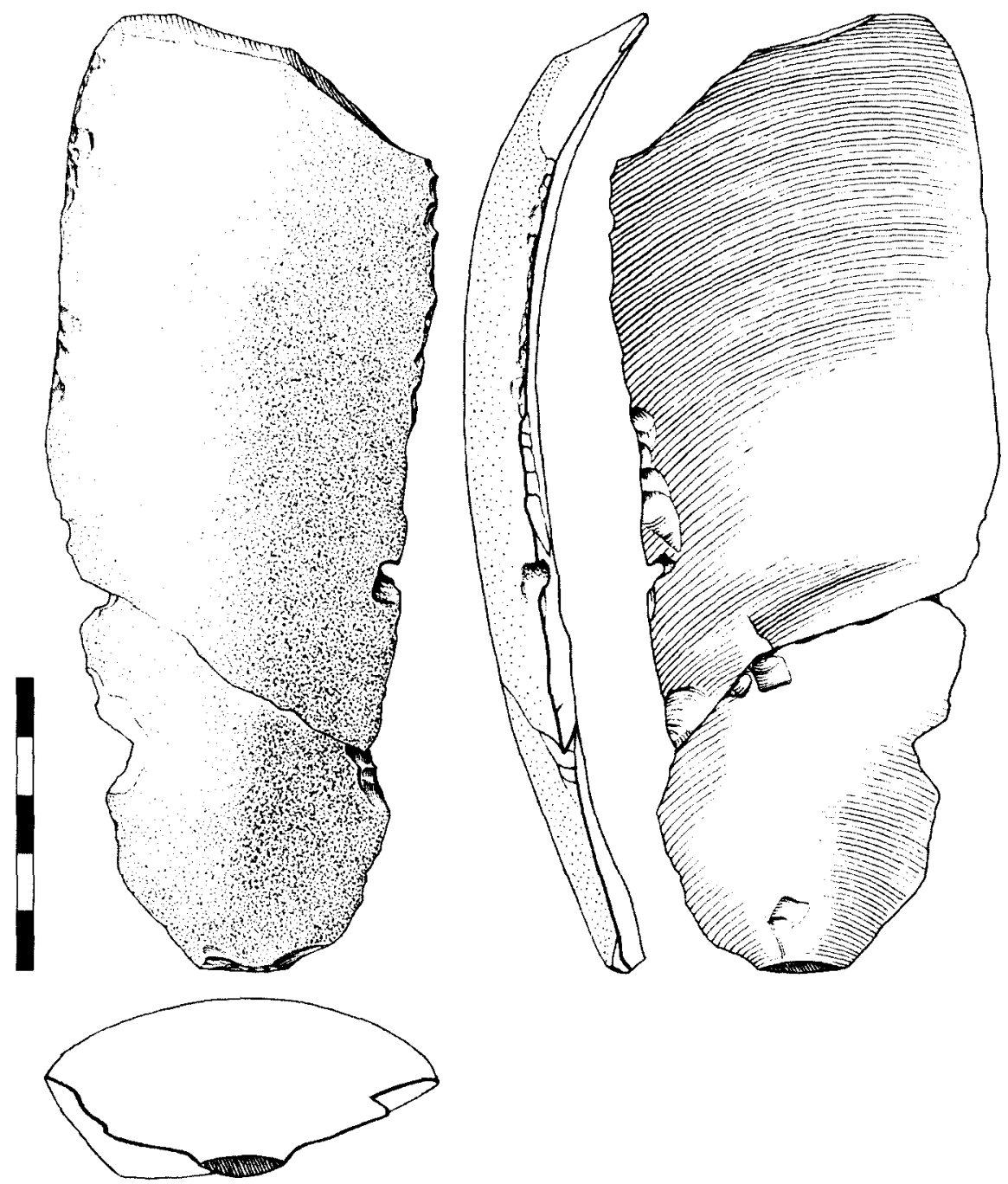

Figure 5. Corbiac-Vignoble II, lame d'entame (le talon est constitué d'une surface naturelle). Dessin J.-G. Marcillaud. 
- plus généralement, les pièces qui ne possèdent aucun tranchant vif et régulier (pas de nervure proéminente le recoupant) sur plus de $5 \mathrm{~cm}$, sur un bord au moins,

- les fragments proximaux et surtout distaux, de profil franchement courbe.

On en déduira que la présence de cortex n'est pas rédhibitoire pour l'emport. On retrouve d'ailleurs fréquemment ce type de support dans les sites d'habitat. Le critère déterminant semble être la présence d'un bord vif (inférieur à 60 degrés), associé à la régularité générale (parallélisme des bords). La partie mésiale des lames régulières, de profil quasi rectiligne, semble préférée aux extrémités plus courbes. Mais si le support est entier, quelle que soit sa courbure, il est emporté.

\subsection{Lamelles}

Les lamelles sont produites à partir de nucléus sur éclat ou lame débités dans leur épaisseur, qui sont classiquement qualifiés de «grattoirs carénés».

\subsubsection{Choix des supports de nucléus, mise en forme}

Les supports des nucléus à lamelles sont exclusivement issus du débitage laminaire effectué sur le site: tous ont pu être remontés ou rapprochés de blocs débités sur place. Ces supports sont, à part égale, des éclats épais et des lames outrepassées (Fig. 3). Ils semblent choisis en fonction de trois caractères au moins:

- un grain particulièrement fin (d'où la grande fréquence de surfaces de débitage implantées dans une zone sous corticale),

- une face inférieure (futur plan de frappe) lisse et régulièrement plane (excluant par exemple la zone bulbaire),

- une épaisseur suffisamment importante pour pouvoir y débiter les lamelles recherchées.

La mise en forme a été décrite par J. Tixier (in Lucas, 1999, pag. 146). Elle consiste en "une sorte de racloir double rectiligne par enlèvements presque abrupts, sans grande application, donnant des négatifs souvent écailleux ou même scalariformes (...)" Des remontages et la présence de plusieurs nucléus abandonnés à différents stades de leur mise en forme confirment pleinement cette description (Fig. 6). 

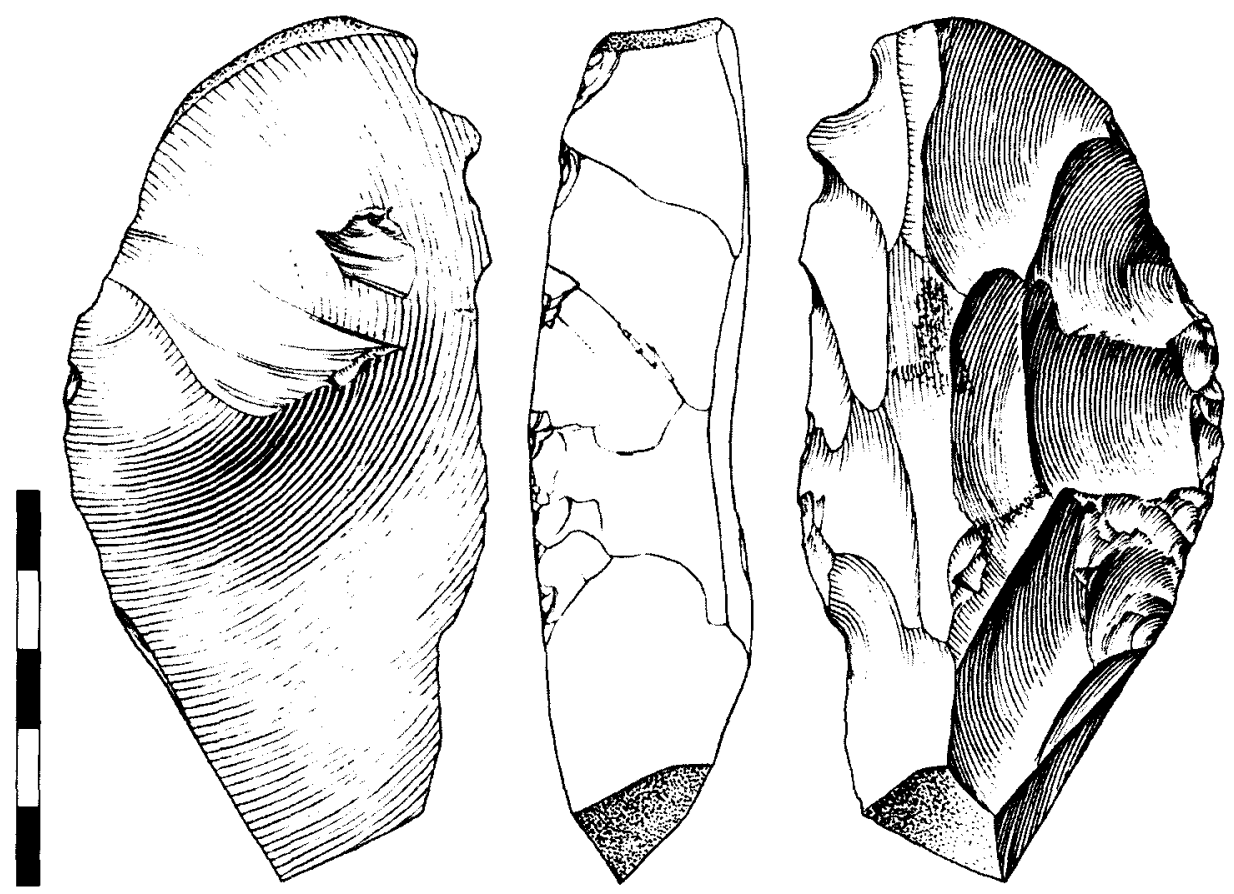

Figure 6. Corbiac-Vignoble II, nucléus à lamelle de type "grattoir caréné" abandonné au stade de sa mise en forme. La zone à débiter se situait en bas, sur le dessin. Dessin J.-G. Marcillaud.

\subsubsection{Plein débitage et entretien}

II existe un continuum depuis les plus épais éclats de coches clactoniennes latérales jusqu'aux fines lamelles de préparation de la surface de débitage. Le détachement des lamelles, effectué à la percussion directe au percuteur tendre, selon un geste très précis et soigneux, est précédé d'une abrasion des corniches laissées par les enlèvements précédents. II est effectué dans l'axe de nervures existantes, ce qui traduit la volonté que le profil ne soit pas tors (Soriano, 1998; Bordes, sous presse). Cette intention est confirmée par l'implantation et la morphologie de la surface de débitage: symétrique par rapport à l'axe morphologique du support; large et ce indépendamment de la présence d'encoches (Figs. 3 et 4). La longueur des lamelles recherchées est comprises entre 2 et $4 \mathrm{~cm}$ de long environ.

Le plan de frappe n'est jamais repris: il reste systématiquement lisse. Parfois, une crête est créee en cours de débitage. Elle sert tant à produire du cintre et de la carène en partie distale de la surface de débitage, qu'à en définir la longueur (Lucas, 1997). 


\subsubsection{Abandon du débitage, abandon des supports}

Contrairement à ce que nous avons constaté pour la production laminaire, pour laquelle la majorité des supports a été exportée, les quelques remontages de séquences lamellaires qui ont pu être "poussés" à leur maximum ne laissent que peu de fantômes de produits emportés. Cela montre que l'image mentale des lamelles recherchées est très précise.

Les raisons de l'abandon de la plupart des nucléus à lamelles ne sont pas évidentes: le débitage semble pouvoir être poursuivi sans modification perceptible des supports produits.

Répétons que les lamelles recherchées sont non torses: celles qui possèdent ce profil, produites fortuitement ou pour aménager la surface de débitage, sont abandonnées sur le site.

Certaines lamelles abandonnées sont très régulières. Un examen plus attentif montre qu'elles ont presque toujours un léger défaut: présence d'une nervure recoupant un des tranchants latéraux, courbure irrégulière (accentuée en partie distale), léger rebroussé... II se peut aussi que de petits objets aient été perdus par les artisans au cours de leur séjour mais cela nous semble peu probable dans la mesure ou il s'agit précisément de l'intention du débitage, auquel un soin particulier a été donné.

\subsection{Dépendance et autonomie des schémas laminaire et lamellaire}

La nature des liens qui unissent les débitages laminaire et lamellaire est un paramètre fondamental de la caractérisation des industries de l'Aurignacien, en particulier pour la distinction entre Proto-Aurignacien ou Aurignacien archaïque du pourtour méditerranéen et Aurignacien ancien (Bon, 2002).

A Corbiac-Vignoble II, il existe d'abord une différence fondamentale autant qu'évidente entre les deux types de supports: le gabarit. Les plus petites lames recherchées font plus de $10 \mathrm{~cm}$ de long, alors que les plus grandes lamelles recherchées ne dépassent pas $4 \mathrm{~cm}$ de long.

Concernant la disjonction technique entre les deux types de production, elle doit être légèrement nuancée. II est vrai que lames et lamelles ne sont pas produites à partir des mêmes nucléus. II reste cependant que dans cet atelier de taille, les deux productions sont effectuées à partir des mêmes blocs. II n'y a donc pas différentiation techno-économique totale entre ces deux productions.

Toutefois, on peut constater que de l'ensemble des blocs débités, il ne semble manquer aucun nucléus à lames. A l'inverse, des indices laissent à croire que des nucléus carénés ont été exportés: fantômes dans des séquences de mise en forme de nucléus à lames, séries d'éclats de coches latérales, non rapprochées 
d'un quelconque nucléus à lamelles présent sur le site. Ces observations gagneraient à être confirmées par la poursuite des remontages. Si elles se vérifiaient, cela irait dans le sens d'une économie un peu différente des productions de lames et des lamelles à l'Aurignacien ancien.

\subsection{Eclats}

Une séquence de production de type Levallois récurrent centripète à été retrouvée dans une zone restreinte du site (un quart de mètre carré), à proximité de l'amas principal. Cette dizaine de pièces qui appartiennent toutes au même bloc (la majorité remontent entre elles) possèdent un état de surface particulier (aspect doux et savonneux) qui ne se retrouve que sur les pièces appartenant au niveau moustérien sous jacent (Tixier et Reduron, 1991). Ces pièces peuvent provenir d'un télescopage des niveaux moustérien et aurignacien à cet endroit, d'un creusement effectué par les aurignaciens jusqu'au niveau moustérien, ou encore d'un phénomène post-dépositionnel naturel qui aurait causé le déplacement vertical d'objets (cryoturbation par exemple). Dans tous les cas, cet ensemble de pièces doit être rapporté au Moustérien sous-jacent.

Hormis ces artefacts, il n'y a aucun nucléus à éclats dans le site.

\subsection{Bilan}

Ces observations, non exhaustives, mériteraient d'être quantifiées pour asseoir des descriptions plus précises. Néanmoins, l'unité technique très forte dont témoigne l'ensemble des nucléus à lames ou à lamelles débités à Corbiac-Vignoble II assure à ces descriptions une valeur générale incontestable: les observations réalisées ici s'organisent toutes en un système technique cohérent, construit autour de deux projets parfaitement maîtrisés par les tailleurs aurignaciens: produire des lames robustes de plus de $10 \mathrm{~cm}$ de long et des lamelles précisément calibrées autour de $2-4 \mathrm{~cm}$ de long.

\section{COMPARAISONS}

Comment replacer Corbiac-Vignoble II dans la séquence aurignacienne? Des rares outils présents (d'ailleurs majoritairement en matières premières non locales), deux pièces orientent l'attribution vers l'Aurignacien ancien: une lame étranglée et une lamelle Dufour de profil non tors (fig. 3b). La méthode de débitage lamellaire mise en œuvre confirme sans ambiguïté ce diagnostic: celle-ci se retrouve systématiquement associée aux sites d'Aurignacien ancien à sagaies à base fendue, à l'exclusion d'une autre phase du technocomplexe aurignacien (Bordes, sous presse). Enfin, le site de Corbiac-Vignoble II constitue un excellent 
complémentaire économique des sites plus classiques dits d'habitat: les supports exportés se retrouvent dans ces derniers sous forme plus ou moins réduite par les différentes opérations de retouche et d'utilisation.

L'ensemble des observations réalisées sur Corbiac-Vignoble II se retrouve dans les gisements que nous avons pu étudier en Dordogne ou dans le Lot. (Caminade-Est $F$ et G, Le Piage F et Gl, Roc-de-Combe 7). Seules trois différences nous semblent devoir être soulignées, qui ne remettent absolument pas en cause l'unité technique décrite ici.

- La consommation des supports est par définition généralisée, et plus poussée. On trouve de fréquents outils sur éclats, supports qui semblent toujours issus de la production laminaire (tablettes de ravivage de plan de frappe, éclats laminaires de recintrage...). On retrouve des lames identiques à celles exportées de Corbiac-Vignoble II, le plus fréquemment sous la forme d'objets les plus caractéristiques de l'équipement des Aurignaciens anciens: grattoirs simples ou doubles sur lames à retouche aurignacienne, burins sur cassure, pièces esquillées... et "grattoirs carénés ".

- La taille des blocs disponibles aux abords de ces gisements, et qui ont parfois contraint les aurignaciens à réaliser des débitages laminaires «en réduction" de ceux de Corbiac-Vignoble II. Ces débitages, s'ils font appel à une plus grande variété de modalités opératoires (par exemple: il n'est pas forcément nécessaire de facetter le plan de frappe sur un nucléus de moins de $10 \mathrm{~cm}$ de long), conservent toujours la même organisation générale.

- Les nucléus à lames sont rares. Ce fait semble pouvoir s'expliquer d'une part par la réduction de la plupart des nucléus présents par un débitage d'éclats sans organisation ni finalité apparentes, et d'autre part par la production des lames hors de ce type de gisement.

Outre les trois gisements précités, tous les sites du nord de l'Aquitaine qui ont fait l'objet d'une analyse technologique récente, et que l'analyse typologique classique avait permis de classer comme appartenant à l'Aurignacien ancien semblent parfaitement s'accorder à la description qui est faite ici (Chadelle 1989, 1990, sous presse; Peyre 1992; Slataper 1995; Pelegrin et O'Farrell, 1998; Chiotti, 1999; Teyssandier 2000; Bon, 2002).

A une échelle géographique plus large, l'Aurignacien ancien tel que défini ici semble se retrouver au moins en Charente (Les Rois, Geoffroy et Mouton, 1958 et obs. pers.; La Quina Aval, Dujardin sous presse); dans les Pyrénées Occidentales et les Cantabres (Arrizabalaga et al., à paraître). D'après F. Bon, il est rare dans le Sud-Est de la France, ne se retrouve pas en Italie sauf à Grimaldi, existe mais reste rare ou à vérifier en Catalogne espagnole (2002, pag. 310 et 322-23). D'après N. Teyssandier (2004, pag. 273), il est présent dans le Jura Souabe, mais réduit à quelques témoignages isolés, voire douteux, sur l'ensemble de l'Europe centrale et dans les Balkans. 


\section{BILAN}

L'Aurignacien aquitain présente, en l'état de nos connaissances, trois phases chronologiques. Encadrée par le ProtoAurignacien ou Aurignacien archaïque à la base, et un Aurignacien récent au sommet, la phase dite ancienne, à retouche aurignacienne fréquente et nucléus à lamelles de type "grattoirs carénés" à front large destinés à produire des lamelles non torses, correspond à l'Aurignacien ancien ou «|», à sagaies à base fendue, de D. Peyrony. C'est l'épisode le plus représenté et le mieux connu de l'Aurignacien aquitain. D'un site à l'autre, les ensembles archéologiques de l'Aurignacien ancien montrent une forte identité des intentions et des modalités des débitages laminaire et lamellaire. Cette unité technique très forte semble construite autour d'une segmentation importante des chaînes opératoires de production lithique, elle-même reflet d'une grande circulation des Hommes et / ou des idées. Ces notions renvoient à l'idée d'une forte prédétermination dans la gestion des ressources lithiques, et témoignent peut-être d'une forte stabilité de l'organisation socio-économique des groupes humains de cette période, plus que jamais repère au sein de l'Aurignacien nordaquitain.

Cette unité a incité de nombreux chercheurs à voir dans cette industrie la trace d'une vague colonisatrice de l'Europe par l'homme anatomiquement moderne. Plusieurs arguments viennent aujourd'hui à l'encontre de cette proposition: 1. l'Aurignacien ancien n'est pas le premier épisode aurignacien du sud-ouest de la France, 2. Son extension est réduite, 3. l'épisode antérieur présente des similitudes avec le Châtelperronien, ce qui contredit l'idée d'une forte rupture entre les industries respectivement attibuées au premiers Hommes antomiquement modernes et aux derniers Néandertaliens d'Europe occidentale. La généralisation des études technologiques, menées aussi bien sur le lithique que sur d'autres catégories de vestiges, devrait permettre d'affiner ce bilan.

\section{BIBLIOGRAPHIE}

Arrizabalaga A., Bon, F., Maillo-Fernández, J.-M., Normand C. \& Ortega, I. (à paraître): Territoires et frontières de l'Aurignacien dans les Pyrénées Occidentales et les Cantabres. In: Cazals dir., Actes de la table ronde de Tarascon, mars 2004.

Bon, F. (2002): L'Aurignacien entre Mer et Océan. Mémoire de la Société Préhistorique Française, $X X \mid X, 253 p$.

Bordes, F. (1958): Le Passage du Paléolithique moyen au Paléolithique supérieur. In: Hundert Jahre neanderthaler: Neanderthal centenary, Utrecht, 1956. Kemink en Zn, p. 175-181.

BORDES, J.-G. (2002): Les interstratifications Châtelperronien / Aurignacien du Roc-de-Combe et du Piage (Lot, France): analyse taphonomique des industries lithiques, implications archéologiques. Thèse de doctorat de l'Universitè Bordeaux 1, $365 \mathrm{p}$.

- (sous presse): Objectifs et modalités de quelques productions lamellaires dans le sud-ouest de la France: Caminade, Roc-de-Combe, Corbiac-Vignoble Il et le Piage. In: F. Le Brun-Ricalens, J.-G. Bordes et F. Bon (Dir.), Productions lamellaires attribuées à l'Aurignacien: chaînes opératoires et perspectives techno-culturelles. Symposium 6-7, XVè Congrès de I'UISPP, Liège, 2-8 Septembre 2001. Université de Liège. 
BORDES, J.-G. et LENOBLE, A. (2002): La «lamelle Caminade», un nouvel outil aurignacien? Bulletin de la Société Préhistorique Française 99(4), p. 735-749.

Bordes, J.-G., Bon, F. et LE BRUN-RICALENS, F. (sous presse): Le transport des matières premières lithiques à l'Aurignacien ancien entre le Nord et Sud de l'Aquitaine: faits attendus, faits nouveaux. $126^{\circ}$ Congrès National des Sociétés historiques et Scientifiques, Toulouse, 9-14 avril 2001.

BrezILlon, M. (1968): La dénomination des objets de pierre taillée. IVè supplément à Gallia Préhistoire, Editions du CNRS, Paris, $425 \mathrm{p}$.

ChADELLE, J.-P. (1989): Les gisements paléolithiques de Champ-Parel, à Bergerac, Dordogne, France. Rapport préliminaire des opérations de sauvetage, 1985-1989. Paléo 1, p. 125-133.

ChADELLE, J.-P. (1990): Le site de plein-air de Corbiac-Vignoble à Bergerac (Dordogne). Technologie lithique et mode d'occuppation. In: Le silex de sa genèse à l'outil, Actes du Vè colloque internationnal sur le silex, Octobre 1987, Cahiers du Quaternaire n. ${ }^{\circ}$ 17, Tome 2, p. 385-390.

- (sous presse): Champarel: un atelier de taille aurignacien en Bergeracois (Dordogne, France). In: Le Brun-Ricalens (Dir.), Productions lamellaires attribuées à l'Aurignacien: chaînes opératoires et perspectives techno-culturelles. Symposium 6-7, Xvè Congrès de I'UISPP, Liège, 2-8 Septembre 2001. Université de Liège.

CHIOTI, L. (1999): Les industries lithiques des niveaux aurignaciens de l'Abri Pataud, les Eyzies-de-Tayac (Dordogne): étude technologique et typologique. Thèse de Doctorat du Muséum d'Histoire Naturelle, Institut de Paléontologie Humaine, Paris, 2t., 839 p.

- (2000): Lamelles Dufour et grattoirs aurignaciens (carénés et à museau) de la couche 8 de l'abri Pataud, Les Eyzies de Tayac, Dordogne. L'Anthropologie 104, p. 239-263.

DEMARS, P.-Y. (1994): L'économie du silex au Paléolithique supérieur dans le Nord de l'Aquitaine. Thèse d'Etat de l'université de Bordeaux I.

Demars, P.-Y. et LAURENT, P. (1989): Types d'outils lithiques du Paléolithique supérieur en Europe. Cahiers du Quaternaire 15. Editions du CNRS, Bordeaux, $178 \mathrm{p}$.

DuJARDIN, V. (sous presse): Débitage lamellaire sur éclat épais à la Quina Aval (Gardes-le-Pontaroux, Charente, France) In: Le Brun-Ricalens (Dir.), Productions lamellaires attribuées à l'Aurignacien: chaines opératoires et perspectives techno-culturelles. Symposium 6-7, Xvè Congrès de l'UISPP, Liège, 2-8 Septembre 2001. Université de Liège.

HaRRolo, F. B. et OTTE, M. (2001): Time, space and cultural proceses in the European Middle-Upper Paleolithic Transition. In: M.A. Hays et P.T. Thacker (Dir.), Questionning the answer: Re-solving Fundamental Problems of the Early Upper Paleolithic., Oxford, BAR International series, 1005, p. 3-12.

KozLOWSKI, J. K. et OTTE, M. (2000): The formation of the Aurignacien in Europe. Journal of Anthropological research 56, p. 513-534.

LE Brun-RICALENS, F. (1989): Contribution à l'étude des pièces esquillées: la présence de pierres à "cupules». Bulletin de la Société Préhistorique française T. 86(7), p. 196-201.

Le Brun-Ricalens, F. et Séronie-Vivien, M. (2004), Paléo, 16, p. 123-136.

LuCAS, G. (1997): Les lamelles Dufour du Flageolet I (Bézenac, Dordogne) dans le contexte aurignacien. Paléo 9, p. 191-219.

- (1999): Production expérimentale de lamelles torses: approche préliminaire. Bulletin de la Société Préhistorique française T. 96(2), p. 145-151.

- (2000): Les industries lithiques du Flageolet / (Dordogne). Approche économique, technologique, fonctionnelle et analyse spatiale. Thèse de l'Université de Bordeaux I, 2. t., $603 \mathrm{p}$.

Mellars, P. (1996): The Neanderthal legacy: an archaeological perspective from western Europe, Princeton, $471 \mathrm{p}$.

- (2004): Neanderthals and the modern human colonization of Europe. Nature 432, p. 461-464.

Mouthon, P. et Joffroy, R. (1958): Le gisement aurignacien des Rois à Mouthiers (Charente). IXéme supplément à Gallia Préhistoire, CNRS Editions. Paris.

Pelegrin, J. (1995): Technologie lithique: le Châtelperronien de Roc de Combe (Lot) et de la Côte (Dordogne). Cahiers du Quaternaire XX, CNRS, Paris.

Pelegrin, J. et O'FarRelL, M. (1998): Quelques éléments sur l'industrie lithique et sur les raccords. In: J. Pelegrin et R. White, Abri Castanet (Sergeac, Dordogne), rapport de fouille programmée triannuelle (1996-1998).

PEYRE, C. (1992): Etude technologique et approche économique du débitage de Champarel locus 3. Mémoire de Maîtrise de l'Université de Paris I, Panthéon -Sorbonne, $91 \mathrm{p}$.

Peyrony, D. (1933): Les industries "aurignaciennes "dans le bassin de la Vézère. Bulletin de la Société Préhistorique Française 30, p.543-559.

- (1934): La Ferrassie: Moustérien, périgordien, Aurignacien. Préhistoire Tome III, p.1-92. 
PIGEOT, N. (1987): Magdaléniens d'Etiolles: économie de débitage et organisation sociale (l'unité d'habitation U5). 25è supplément à Gallia Préhistoire, CNRS, Paris.

Ploux, S. et SORIANO, S (2003): Umm El Tlel, une séquence du Paléolithique supérieur en Syrie centrale. Industries lithiques et chronologie culturelle. Paléorient 29(2), p. 5-34.

Slataper, M. (1995): Analyse des méthodes de débitage de l'Aurignacien. Premiers résultats de l'approche technologique d'un amas de taille: la concentration C5 de Barbas III. In Mémoire de DEA de l'Université de PAris I, Panthéon-Sorbonne, $102 \mathrm{p}$.

SONNEVILle-BORdes, D. de (1960): Le Paléolithique supérieur en Périgord. Bordeaux, imprimerie Delmas, 2 vol, $580 \mathrm{p}$.

TEYSSANDIER, N. (2000): L'industrie lithique aurignacienne du secteur II de Barbas (Creysse, Dordogne). Analyse technique et implications archéologiques. In: E. Boëda et V. Guillomet-Malmassari (Dir.) Des comportements techniques dans la Préhistoire, Nanterre, p. 29-59.

- (2003): Les débuts de l'Aurignacien en Europe. Discussion à partir des sites de Geissenklösterle, Willendorf II, Krems-hundssteig et Bacho-Kiro. Thèse de l'Université de Paris X-Nanterre, $326 \mathrm{p}$.

TIXIER, J. (1991): Champ-Parel, Corbiac-Vignoble 2 (CV2). Gallia informatioins, Aquitaine, Editions du C.N.R.S., p. 8-9.

TIXIER, J. et REDURON, M. (1991): Et passez au pays des silex: rapportez-nous des lames ! In: 25 ans d'études technologiques en Préhistoire. Xlè Rencontres Internationales d'Archéologie et d'Histoire d'Antibes, p. 235-243. 